Annals of Pure and Applied Mathematics

Vol. 15, No. 2, 2017, 209-214

ISSN: 2279-087X (P), 2279-0888(online)

Published on 11 December 2017

www.researchmathsci.org

DOI: http://dx.doi.org/10.22457/apam.v15n2a7

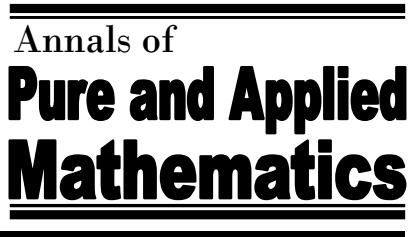

\title{
Analytic Solution of Linear Fractional Differential Equation Using Fractional Laplace Transform
}

\author{
S. Ruban Raj ${ }^{1}$ and S. Sahaya Jernith ${ }^{2}$ \\ ${ }^{1}$ Department of Mathematics, St.Joseph's College \\ Trichy, Tamilnadu, India. \\ ${ }^{2}$ Research scholar, Department of Mathematics \\ St. Joseph's College, Trichy, Tamilnadu, India. \\ Received 1 November 2017; accepted 5 December 2017
}

Abstract. In this paper we present some properties in order to solve certain fractional differential equation. Mittag-Leffler function plays an important role in the Laplace transform to find the solution of fractional order differential equations. Here we solve some problems related to fractional order differential equation using fractional Laplace Transform with initial conditions.

Keywords: Riemann-Liouville derivative, Mittag-Leffler function, Jumarie Derivative, Fractional Laplace Transforms, Fractional differential equations.

\section{AMS Mathematics Subject Classification (2010): 34A08}

\section{Introduction}

Fractional calculus arises from a question posed by L'Hospital and Lebnitz in1965. It is the generalization of integer-order calculus. Reviewing the history we find that the Fractional calculus was more interesting topic to mathematicians for a long time in spite of the lack of application back ground. Upcoming years more and more researchers have paid their attention towards Fractional Calculus which are used in real world problems such as Viscoelastic system, dielectric polarization, electromagnetic waves, etc., [8, 9, 5, 7]. With the great efforts of researchers there have been rapid developments on the theory of fractional calculus and its applications. The purpose of this paper is to define the Fractional Laplace Transform via Mittag-Leffler function. Mittag-Leffler function is defined and with its help the Fractional Laplace Transform is also defined. Here we derive some properties of Fractional Laplace Transform based on our definition which is more helpful to find the solution of fractional differential equation with the initial conditions.

\section{Preliminaries}

Definition 2.1. (Riemann-Liouville definition of fractional derivative). Let the function $\mathrm{g}(\mathrm{t})$ be one time integrable. Then the integro-differential defines Riemann-Liouville fractional derivative[2]

$$
{ }_{b} D_{t}^{\alpha}[g(t)]=\frac{1}{\Gamma(n-\alpha)}\left(\frac{d}{d t}\right)^{n} \int_{b}^{t}(t-\tau)^{(n-\alpha)-1} g(\tau) d \tau, \alpha>0
$$




\section{S. Ruban Raj and S. Sahaya Jernith}

This expression is known as the Riemann-Liouville definition of fractional derivative. By this definition, fractional derivative of a constant is non-zero.

Definition 2.2. (Modified Riemann-Liouville $(\mathrm{RL})$ ). To overcome the shortcoming that the fractional derivative of a constant is non-zero, the modification in the definition of the fractional derivative, proposed by Jumarie [5] is described as below:

$$
D_{t}^{\alpha}[g(t)]=\left\{\begin{array}{l}
\frac{1}{\Gamma(-\alpha)} \int_{b}^{t}(t-\tau)^{-\alpha-1} f(\tau) d \tau, \alpha<0 \\
\frac{1}{\Gamma(1-\alpha)} \frac{d}{d x} \int_{b}^{t}(t-\tau)^{-\alpha}[f(\tau)-f(b)] d \tau, 0<\alpha<1 \\
\left(f^{(\alpha-m)}(x)\right)^{m}, m \leq \alpha<m+1
\end{array}\right.
$$

Definition 2.3. (Mittag-Leffler Function). The one-parameter of Mittag-Leffler function [3], denoted by $\mathrm{E}_{\alpha}(\mathrm{z})$, is defined by

$$
E_{\alpha}(z)=\sum_{k=0}^{\infty} \frac{z^{k}}{\Gamma(1+\alpha k)}, z \in \mathbb{C}, \operatorname{Re}(\alpha)>0
$$

\subsection{Mittag-Leffler function for fractional derivative}

Mittag-Leffler function is defined in the form of an infinite series with one parameter [3]

$$
E_{\beta}\left(\alpha x^{\beta}\right)=1+\frac{\alpha x^{\beta}}{\Gamma(1+\beta)}+\frac{\alpha^{2} x^{2 \beta}}{\Gamma(1+2 \beta)}+\frac{\alpha^{3} x^{3 \beta}}{\Gamma(1+3 \beta)}+\cdots \infty
$$

Definition 2.4. (Fractional Derivative of Mittag-Leffler Function). The Jumarie derivative [3] of the Mittag-Leffler function $\mathrm{E}_{\beta}\left(\alpha \mathrm{x}^{\beta}\right)$ is defined as follows:

Applying term by term Modified RL derivative we get,

$$
\begin{aligned}
D^{\beta}\left[E_{\beta}\left(\alpha x^{\beta}\right)\right] & =D^{\beta}\left[1+\frac{\alpha x^{\beta}}{\Gamma(1+\beta)}+\frac{\alpha^{2} x^{2 \beta}}{\Gamma(1+2 \beta)}+\frac{\alpha^{3} x^{3 \beta}}{\Gamma(1+3 \beta)}+\cdots \infty\right] \\
& =\alpha\left[1+\frac{\alpha x^{\beta}}{\Gamma(1+\beta)}+\frac{\alpha^{2} x^{2 \beta}}{\Gamma(1+2 \beta)}+\frac{\alpha^{3} x^{3 \beta}}{\Gamma(1+3 \beta)}+\cdots \infty\right] \\
& =\alpha E_{\beta}\left(\alpha x^{\beta}\right)
\end{aligned}
$$

where $\beta$ is the order of the Jumarie derivative of Mittag-Leffler function.

Definition 2.5. (Fractional Laplace Transform) If a function $f(t)$ is defined for all positive values of the variable $t$ and if $\int_{0}^{\infty} E_{\beta}\left(-s^{\beta} t^{\beta}\right) f(t)(d t)^{\beta}$ exists and is equal to (s), then $F(s)$ is called the Fractional Laplace Transform [4] of $f(t)$, denoted by the symbol $\mathrm{L}_{\beta}[\mathrm{f}(\mathrm{t})]$. Hence

$$
\mathrm{L}_{\beta}[\mathrm{f}(\mathrm{t})]=\int_{0}^{\infty} \mathrm{E}_{\beta}\left(-\mathrm{s}^{\beta} \mathrm{t}^{\beta}\right) \mathrm{f}(\mathrm{t})(\mathrm{dt})^{\beta}=\mathrm{F}(\mathrm{s})
$$

The operator $\mathrm{L}_{\beta}$ that transforms $\mathrm{f}(\mathrm{t})$ into $\mathrm{F}(\mathrm{s})$ is called the Fractional Laplace transform operator. 
Analytic Solution of Linear Fractional Differential Equation Using Fractional Laplace Transform

\section{Main results}

Property 3.1. $L_{\beta}[f(t)+g(t)]=L_{\beta}[f(t)]+L_{\beta}[g(t)]$.

\section{Proof:}

$$
\begin{aligned}
L_{\beta}[f(t)+g(t)]=\int_{0}^{\infty} E_{\beta} & \left(-s^{\beta} t^{\beta}\right)[f(t)+g(t)](d t)^{\beta} \\
= & \int_{0}^{\infty} E_{\beta}\left(-s^{\beta} t^{\beta}\right) f(t)(d t)^{\beta}+\int_{0}^{\infty} E_{\beta}\left(-s^{\beta} t^{\beta}\right) g(t)(d t)^{\beta} \\
= & L_{\beta}[f(t)]+L_{\beta}[g(t)]
\end{aligned}
$$

Property 3.2. $L_{\beta}[c f(t)]=c L_{\beta}[f(t)]$, where $c$ is a constant.

\section{Proof:}

$$
\begin{aligned}
L_{\beta}[c f(t)] & =\int_{0}^{\infty} E_{\beta}\left(-s^{\beta} t^{\beta}\right) c f(t)(d t)^{\beta} \\
& =c \int_{0}^{\infty} E_{\beta}\left(-s^{\beta} t^{\beta}\right) f(t)(d t)^{\beta} \\
& =c L_{\beta}[f(t)]
\end{aligned}
$$

Property 3.3. $. L_{\beta}\left[t^{\beta n}\right]=\frac{\Gamma(n \beta+1)}{s^{n \beta+\beta}}$.

Proof:

put $s^{\beta} t^{\beta}=x^{\beta}$. Then

$$
\mathrm{L}_{\beta}\left[\mathrm{t}^{\beta \mathrm{n}}\right]=\int_{0}^{\infty} \mathrm{E}_{\beta}\left(-\mathrm{s}^{\beta} \mathrm{t}^{\beta}\right) \mathrm{t}^{\beta \mathrm{n}}(\mathrm{dt})^{\beta}
$$

$\mathrm{L}_{\beta}\left[\mathrm{t}^{\beta \mathrm{n}}\right] \quad=\frac{(\mathrm{n} \beta) !}{\mathrm{s}^{\mathrm{n} \beta+\beta}}$

$$
\begin{aligned}
\mathrm{L}_{\beta}\left[\mathrm{t}^{\beta \mathrm{n}}\right] & =\int_{0}^{\infty}\left(\frac{\mathrm{x}}{\mathrm{s}}\right)^{\mathrm{n} \beta} \mathrm{E}_{\beta}\left(-\mathrm{x}^{\beta}\right) \frac{1}{\mathrm{~s}^{\beta}}(\mathrm{dx})^{\beta} \\
& =\frac{1}{\mathrm{~s}^{\mathrm{n} \beta+\beta}} \int_{0}^{\infty} \mathrm{x}^{\mathrm{n} \beta} \mathrm{E}_{\beta}\left(-\mathrm{x}^{\beta}\right)(d x)^{\beta} \\
& =\frac{1}{\mathrm{~s}^{\mathrm{n} \beta+\beta}}(\mathrm{n} \beta) !
\end{aligned}
$$

Property 3.4. $L_{\beta}\left[E_{\beta}\left(a^{\beta}\right)\right]=\frac{1}{s^{\beta}-a}, s^{\beta} \neq a$.

Proof:

$$
\begin{aligned}
\mathrm{L}_{\beta}\left[\mathrm{E}_{\beta}\left(\mathrm{at}^{\beta}\right)\right] & =\mathrm{L}_{\beta}\left[\sum_{\mathrm{n}=0}^{\infty} \frac{\mathrm{a}^{\mathrm{n}} \mathrm{t}^{\beta \mathrm{n}}}{\Gamma(\beta \mathrm{n}+1)}\right]=\sum_{\mathrm{n}=0}^{\infty} \frac{\mathrm{a}^{\mathrm{n}}}{\Gamma(\beta \mathrm{n}+1)} \mathrm{L}_{\beta}\left[\mathrm{t}^{\beta \mathrm{n}}\right] \\
& =\sum_{\mathrm{n}=0}^{\infty} \frac{\mathrm{a}^{\mathrm{n}}}{\Gamma(\beta \mathrm{n}+1)} \frac{\Gamma(1+\beta \mathrm{n})}{\mathrm{s}^{\mathrm{n} \beta+\beta}} \\
& =\sum_{\mathrm{n}=0}^{\infty} \frac{\mathrm{a}^{\mathrm{n}}}{\Gamma(\beta \mathrm{n}+1)} \frac{\Gamma(1+\beta \mathrm{n})}{\mathrm{s}^{\mathrm{n} \beta+\beta}}
\end{aligned}
$$


S. Ruban Raj and S. Sahaya Jernith

$$
\begin{aligned}
& =\frac{1}{s^{\beta}} \sum_{n=0}^{\infty}\left(\frac{a}{s^{\beta}}\right)^{n} \\
& =\frac{1}{s^{\beta}-a}
\end{aligned}
$$

Property 3.5. $\mathrm{L}_{\beta}\left(D^{\beta} \mathrm{f}(\mathrm{t})\right)=\mathrm{s}^{\beta} \mathrm{L}_{\beta}(\mathrm{f}(\mathrm{t}))-\mathrm{f}(0)$.

Proof:

$$
\begin{aligned}
& L_{\beta}\left(D^{\beta} f(t)\right)=\int_{0}^{\infty} E_{\beta}\left(-s^{\beta} t^{\beta}\right)\left(D^{\beta} f(t)\right)(d t)^{\beta} \\
= & {\left[f(t) E_{\beta}\left(-s^{\beta} t^{\beta}\right)\right]_{0}^{\infty}-\int_{0}^{\infty} f(t)\left(-s^{\beta} E_{\beta}\left(-s^{\beta} t^{\beta}\right)\right)(d t)^{\beta} } \\
= & -f(0)+s^{\beta} \int_{0}^{\infty} f(t)\left(E_{\beta}\left(-s^{\beta} t^{\beta}\right)\right)(d t)^{\beta} \\
= & -f(0)+s^{\beta} L_{\beta}(f(t)) \\
= & s^{\beta} L_{\beta}(f(t))-f(0) .
\end{aligned}
$$

Property 3.6. $L_{\beta}\left(D^{2 \beta} f(t)\right)=s^{2 \beta} L_{\beta}(f(t))-s^{\beta} f\left((0)-f^{\beta}(0)\right.$.

Proof:

$$
L_{\beta}\left(D^{2 \beta} f(t)\right)=s^{2 \beta} L_{\beta}(f(t))-s^{\beta} f(0)-f^{\beta}(0)
$$

$\mathrm{L}_{\beta}\left(\mathrm{f}^{2 \beta}(\mathrm{t})\right)=\mathrm{L}_{\beta}\left(D^{\beta} \mathrm{F}(\mathrm{t})\right)$ whereF $(\mathrm{t})=\mathrm{f}^{\beta}(\mathrm{t})$

$$
\begin{aligned}
= & s^{\beta} L_{\beta}(F(t))-F(0) \\
= & s^{\beta} L_{\beta}\left(f^{\beta}(t)\right)-f^{\beta}(0) \\
= & s^{\beta}\left[s^{\beta} L_{\beta} f(t)-f(0)\right]-f^{\beta}(0) \\
= & s^{2 \beta} L_{\beta} f(t)-s^{\beta} f(0)-f^{\beta}(0)
\end{aligned}
$$

By extending the result, we get

$$
L_{\beta}\left[f^{(n \beta)}(t)\right]=s^{(n \beta)} L_{\beta}[f(t)]-s^{(n \beta-\beta)} f(0)-s^{(n \beta-2 \beta)} f^{(\beta)}(0)-s^{(n \beta-3 \beta)} f^{(2 \beta)}(0)-
$$
$\ldots-\mathrm{f}^{(\mathrm{n} \beta-\beta)}(0)$

Property 3.7. $\mathrm{L}_{\beta}\left(\cos _{\beta}\left(\mathrm{at}{ }^{\beta}\right)\right)=\frac{\mathrm{s}^{\beta}}{\mathrm{s}^{2 \beta}+\mathrm{a}^{2}}$.

Proof:

$$
\begin{gathered}
f(t)=\cos _{\beta}\left(a t^{\beta}\right) \\
f^{\beta}(t)=-\operatorname{asin}_{\beta}\left(a t^{\beta}\right) \\
f^{2 \beta}(t)=-a^{2} \cos _{\beta}\left(a t^{\beta}\right) \\
L_{\beta}\left(D^{2 \beta} f(t)\right)=s^{2 \beta} L_{\beta} f(t)-s^{\beta} f(0)-f^{\beta}(0) \\
L_{\beta}\left(-a^{2} \cos _{\beta}\left(a t^{\beta}\right)\right)=s^{2 \beta} L_{\beta}\left[\cos _{\beta}\left(a t^{\beta}\right)\right]-s^{\beta} \\
s^{\beta}=\left(s^{2 \beta}+a^{2}\right) L_{\beta}\left(\cos _{\beta}\left(a t^{\beta}\right)\right) \\
L_{\beta}\left(\cos _{\beta}\left(a t^{\beta}\right)\right)=s^{2 \beta}+a^{2}
\end{gathered}
$$

Property 3.8. $\mathrm{L}_{\beta}\left(\sin _{\beta}\left(\mathrm{at}^{\beta}\right)\right)=\frac{\mathrm{a}}{\mathrm{s}^{2 \beta}+\mathrm{a}^{2}}$. 
Analytic Solution of Linear Fractional Differential Equation Using Fractional Laplace

Transform

\section{Proof:}

$$
\begin{aligned}
& \mathrm{f}(\mathrm{t})=\sin _{\beta}\left(a \mathrm{t}^{\beta}\right) \\
& f^{\beta}(t)=-\operatorname{acos}_{\beta}\left(a t^{\beta}\right) \\
& \mathrm{f}^{2 \beta}(\mathrm{t})=-\mathrm{a}^{2} \sin _{\beta}\left(\mathrm{at}^{\beta}\right) \\
& \mathrm{L}_{\beta}\left(D^{2 \beta} \mathrm{f}(\mathrm{t})\right)=\mathrm{s}^{2 \beta} \mathrm{L}_{\beta} \mathrm{f}(\mathrm{t})-\mathrm{s}^{\beta} \mathrm{f}(0)-\mathrm{f}^{\beta}(0) \\
& \mathrm{L}_{\beta}\left[\sin _{\beta}\left(\mathrm{at}^{\beta}\right)\right]=\frac{\mathrm{a}}{\mathrm{s}^{2} \mathrm{a}^{2}} \text {. } \\
& \left.a^{2} \sin _{\beta}\left(a^{\beta}\right)\right)=s^{2 \beta} L_{\beta}\left[\sin _{\beta}\left(a^{\beta}\right)\right]-0-a
\end{aligned}
$$

Property 3.9. $\mathrm{L}_{\beta}\left(\mathrm{E}_{\beta}\left(-a t^{\beta} \mathrm{f}(\mathrm{t})\right)\right)=\mathrm{F}\left(\mathrm{s}^{\beta}+\mathrm{a}\right)$.

Proof: We know that, $\mathrm{F}(\mathrm{s})=\mathrm{L}_{\beta}[\mathrm{f}(\mathrm{t})]=\int_{0}^{\infty} \mathrm{E}_{\beta}\left(\mathrm{s}^{\beta} \mathrm{t}^{\beta}\right) \mathrm{f}(\mathrm{t})(\mathrm{dt})^{\beta}$

$$
\begin{aligned}
\mathrm{L}_{\beta}\left[\mathrm{E}_{\beta}\left(-\mathrm{at}^{\beta}\right)\right] & =\int_{0}^{\infty} \mathrm{E}_{\beta}\left(-\mathrm{s}^{\beta} \mathrm{t}^{\beta}\right) \mathrm{E}_{\beta}\left(-\mathrm{at} \mathrm{t}^{\beta}\right) \mathrm{f}(\mathrm{t})(\mathrm{dt})^{\beta} \\
& =\int_{0}^{\infty} \mathrm{E}_{\beta}\left(-\mathrm{s}^{\beta}+\mathrm{a}\right) \mathrm{t}^{\beta} \mathrm{f}(\mathrm{t})(\mathrm{dt})^{\beta} \\
& =\mathrm{F}\left(\mathrm{s}^{\beta}+\mathrm{a}\right)
\end{aligned}
$$

Example 3.10. We solve the following homogeneous FDE using Fractional Laplace Transform, $\left(D^{2\left(\frac{1}{4}\right)}+2 D^{\frac{1}{4}}+2\right) y(t)=0$ where $y(0)=1, y^{\left(\frac{1}{4}\right)}(0)=-1$

Solution: The equation can be written in the form

Applying fractional Laplace transforms to both sides, we have

$$
\mathrm{y}^{2\left(\frac{1}{4}\right)}+2 \mathrm{y}^{\left(\frac{1}{4}\right)}+2 \mathrm{y}=0
$$

$$
\begin{gathered}
\mathrm{L}_{\frac{1}{4}}\left[\mathrm{y}^{2\left(\frac{1}{4}\right)}\right]+2 \mathrm{~L}_{\frac{1}{4}}\left[\mathrm{y}^{\left(\frac{1}{4}\right)}\right]+2 \mathrm{~L}_{\frac{1}{4}}[\mathrm{y}]=0 \\
\mathrm{~s}^{\frac{2}{4}} \mathrm{~L}_{\frac{1}{4}}[\mathrm{y}(0)]-\mathrm{s}^{\frac{1}{4}} \mathrm{y}(0)-\mathrm{y}^{\left(\frac{1}{4}\right)}(0)+2\left[\mathrm{~s}^{\frac{1}{4}} \mathrm{~L}_{\frac{1}{4}}[\mathrm{y}(0)]-\mathrm{y}(0)\right]+2 \mathrm{~L}_{\frac{1}{4}}[\mathrm{y}(0)]=0 \\
\left.\mathrm{~s}^{\frac{2}{4}}+2 \mathrm{~s}^{\frac{1}{4}}+2\right) \mathrm{L}_{\frac{1}{4}}[\mathrm{y}(\mathrm{t})]=\mathrm{s}^{\frac{1}{4}}+1 \\
\mathrm{~L}_{\frac{1}{4}}[\mathrm{y}(\mathrm{t})]=\frac{\mathrm{s}^{\frac{1}{4}}+1}{\mathrm{~s}^{\frac{2}{4}}+2 \mathrm{~s}^{\frac{1}{4}}+2} \\
\mathrm{~s}(\mathrm{t})]=\frac{\mathrm{s}^{\frac{1}{4}}+1}{\left(\mathrm{~s}^{\frac{1}{4}}+1\right)^{2}+2}=\mathrm{E}_{\frac{1}{4}}\left(-\mathrm{t}^{\frac{1}{4}}\right) \cos _{\frac{1}{4}}\left(\mathrm{t}^{\frac{1}{4}}\right)
\end{gathered}
$$

\section{Conclusion}

The Laplace transformation method has been successfully applied to find an exact solution of Fractional Differential Equation. Some results are derived with the proofs. We 


\section{S. Ruban Raj and S. Sahaya Jernith}

conclude that the Laplace transformation method is a powerful efficient tool for finding a solution of Fractional Differential Equation.

\section{REFERENCES}

1. S.Das, Functional Fractional Calculus, 2nd Edition, Springer-Verlag, Germany

2. K. Diethelm, The analysis of Fractional Differential Equations, Springer-Verlag, Germany (2010).

3. Guy Jumarie, Laplace's transform of fractional order via the mittag-Leffler function and modified Riemann-Liouville derivative, Applied Mathematics Letters, 22 (2009) $1659-1664$.

4. A.Kilbas, H.M.Srivastave, J.J.Trujillo, Theory and Applications of Fractional Differential Equations, Northern-Holland Mathematics Studies, Elsevier Science, Amsterrdam the Netherlands (2006).

5. K.S.Miller, B.Ross, An Introduction to the Fractional Calculus and Fractional Differential Equations, Jonh Wiley \& Sons, New York, NY, USA (1993).

6. I.Podlubny, Fractional differential equations: An introduction to fractional derivatives, fractional differential equations, to methods of their solution and some of their application, Academic Press, San Diego, CA, 1999.

7. G.C.Wu, E.W.M.Lee, Fractional Varational Iteration Method and its Applications, Phys. Lett. A. (2010).

8. S. Zhang and H.Q. Zhang, Fractional Sub-equation Method and its applications to nonlinear fractional PDES, Phys. Lett. A. (2011). 